\title{
Diffractive beamshaping elements at the fabrication limit
}

\author{
Wolfgang Singer \\ Institut fuer Technische Optik \\ Pfaffenwaldring 9 \\ D-70569 Stuttgart, Germany \\ E-mail: singer@ito311.ito.uni-stuttgart.de \\ Hans Peter Herzig \\ Institut de Microtechnique \\ Rue A.-L. Breguet 2 \\ $\mathrm{CH}-2000$ Neuchâtel, Switzerland \\ Markku Kuittinen \\ University of Joensuu \\ Department of Physics \\ Väisälä Laboratory \\ P.O. Box 111 \\ FIN-80101 Joensuu, Finland \\ Eckhard Piper \\ Johannes Wangler \\ Carl Zeiss \\ D-73446 Oberkochen, Germany
}

\section{Introduction}

Diffractive micro-optical elements have gained increasing interest for beam shaping, e.g., the laser treatment of materials ${ }^{1}$ or for illumination systems. The fabrication technologies for diffractive micro-optical elements have brought about an advance high accuracy and reproducibility, especially of the periodicity of the elements. In monochromatic applications, diffractive micro-optical elements are restricted only by the limits of the fabrication technology. Beside these restrictions, diffractive optical elements are able to fulfill almost any requirements for monochromatic applications.

In this paper, we discuss the design of optical diffusors for the fabrication of flat-top intensity distributions, ${ }^{2}$ as required for 248-nm excimer laser applications. We investigated far-field distributions with either rectangular or circular shapes with uniform intensity and high efficiency. For the application in illumination systems, as in lithography, a space-invariant response of the diffractive elements is required. In detail, the diffusors must be almost independent of the size, the shape, and the homogenity of the illumination. For a practical application in flexible systems, the elements should furthermore be insensitive to small alignment errors.

The fabrication limits due to the restricted resolution of the fabrication technology are a severe restriction for the design of diffractive elements for applications at small wavelengths. Therefore a conventional design was preferred to the iterative Fourier transform algorithm ${ }^{3}$ (IFTA). The utilization of arrays of binary micro-Fresnel lenses ${ }^{4}$ with adapted geometry is straightforward for this application. The lenses generate the desired angular spectrum, while the array property warrants the space invariance of the element. However, binary Fresnel lenses suffer usually from binarization noise and a large zeroth diffraction order, which violates the homogenity condition of flat-top distributions. The small oscillations in the far field due to the binarization noise can be smoothed out with partially coherent or, in the limit, incoherent illumination. Furthermore we show that the zeroth order is reduced by the addition of a constant phase offset to the transmission phase function of the lens array.

Binary diffractive elements with only two phase levels usually have a low diffraction efficiency ${ }^{5}(40.5 \%)$. For the generation of symmetrical intensity distributions, however, both the plus and the minus first diffraction orders contribute to the desired intensity distribution, increasing the diffraction efficiency $(\sim 80 \%)$. To improve the efficiency, multilevel elements must be employed. Therefore we also investigate elements with varying numbers of phase levels. The number of levels is thereby choosen according to the resolution limit of the fabrication technology. As is shown, these elements have a locally varying diffraction efficiency, giving rise to an inhomogeneous intensity distribution. Therefore, for large diffraction angles at small wavelengths, binary elements are favorable.

In Section 2, we introduce our design and explain the optimization method for the lenses. In Section 3, we describe the calculation method for the far field. Section 4 treats the effect of an offset phase to the far field of binaryand multilevel phase gratings. Section 5 summarizes the results for binary elements. The results are further compared to measurements. The effect of fabrication errors is 
Singer et al.: Diffractive beamshaping elements...

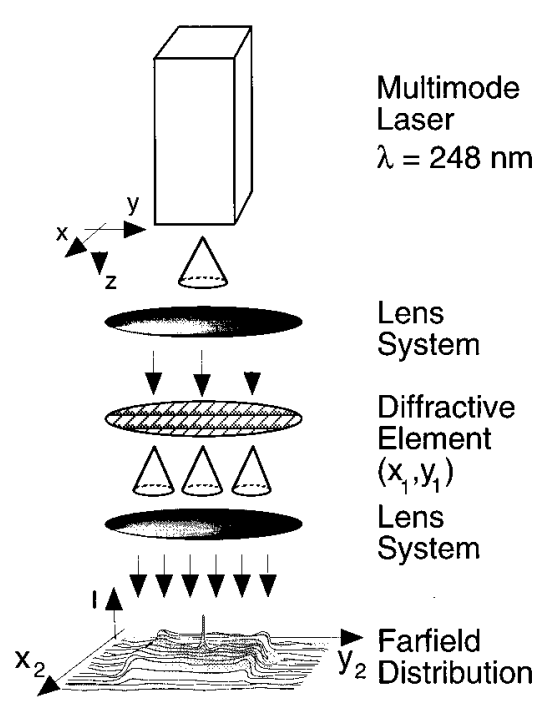

Fig. 1 Set-up for the generation of flat-top distributions (simplified).

treated in Section 6. A short discussion of multilevel elements follows in Section 7.

\section{Design Considerations}

In our investigation, the following setup was considered: a diffractive element is illuminated by a multi-mode excimer laser with given beam divergency in $x$ and $y$ direction at a wavelength $\lambda=248 \mathrm{~nm}$. The required intensity distribution is then obtained in the Fourier plane of the second lens system (Fig. 1).

The phase grating to generate the flat-top intensity distribution is designed as an array of micro-Fresnel lenses of hexagonal or rectangular shape. For a given focal length, the shape of a single diffractive lens defines the shape and the diameter $D$ of the far-field distribution. Due to the divergent illumination with an incoherent source, the far field of the lenses is added incoherently and with small displacements. This can also be described in conventional Fourier optics by the use of an extended source $U_{0}\left(x_{0}, y_{0}\right)$ in the Fourier domain, where the extension $S$ of the source is defined by the divergency angle (Fig. 2). ${ }^{6}$

The diffractive element with the size $L X$ and $L Y$ is considered in the thin-element approximation by a complex transmission function ${ }^{7} T_{1}$ :

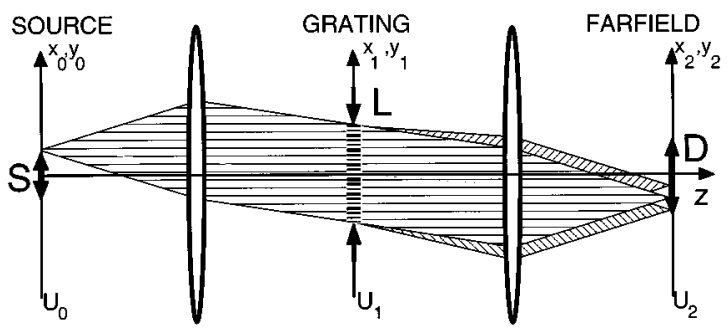

Fig. 2 Design considerations and coordinates.
$T_{1}\left(x_{1}, y_{1}\right)=\left\{\begin{array}{l}0 \\ \exp \left[i \varphi\left(x_{1}, y_{1}\right)\right]\end{array}\right.$ for $\begin{array}{ll}x_{1} \geqslant L X, & y_{1} \geqslant L Y \\ x_{1}<L X, & y_{1}<L Y .\end{array}$

In the case of coherent illumination, the intensity distribution $I_{2}$ in the far field is given by a convolution of the amplitudes

$$
\begin{aligned}
I_{2}\left(x_{2}, y_{2}\right) & =\left|U_{2}\left(x_{2}, y_{2}\right)\right|^{2} \\
& =\left|\operatorname{FT}\left[U_{1}\left(x_{1}, y_{1}\right)\right]\right|^{2} \\
& =\left|\operatorname{FT}\left\{\mathrm{FT}^{-1}\left[U_{0}\left(x_{0}, y_{0}\right)\right] T_{1}\left(x_{1}, y_{1}\right)\right\}\right|^{2} \\
& =\left|U_{0}\left(x_{0}, y_{0}\right) \otimes \operatorname{FT}\left[T_{1}\left(x_{1}, y_{1}\right)\right]\right|^{2} .
\end{aligned}
$$

In the case of incoherent illumination, however, the intensity distribution $I_{2}$ in the far field is given by a convolution of the intensities

$I_{2}\left(x_{2}, y_{2}\right)=\left|U_{0}\left(x_{0}, y_{0}\right)\right|^{2} \otimes\left|\mathrm{FT}\left[T_{1}\left(x_{1}, y_{1}\right)\right]\right|^{2}$.

In the following we assume that the source is ideally incoherent. The modal distribution of the laser is approximated very roughly by a rect function. The convolution of the intensity distribution with a rect function corresponds to a detection of the intensity distribution with a common detector with a finite pixel size. This must be taken into account for a comparison of the far-field distribution with measurements. As is shown in detail, the incoherent source has a smoothing effect on the fine oscillations of the coherent intensity distribution.

\section{Calculation Methods for the Far-Field Distribution}

For the solution of the problem according to Eq. (3), a Fourier transformation (FT) is required. In one dimension, this can easily be performed by a fast fourier transformation (FFT) algorithm. ${ }^{8}$ However, for small wavelengths (e.g., $\lambda=248 \mathrm{~nm}$ ) and large dimensions of the single lenses (e.g., $\sim 1000 \mu \mathrm{m}$ ) of the array it is impossible to extend the calculation to the 2-D case. Because we are concerned only with distributions of rotational symmetry, the Hankel transformation ${ }^{9}$ is applicable.

However, phase gratings or Fresnel zone lenses are conventionally fabricated as a binary or multilevel phase grating. In that case, the direct computation of the far field according to the Fresnel integral by a sum of Bessel functions is preferable. ${ }^{10}$ This algorithm is also applicable for one dimension, resulting in a sum of sinc functions. For this reason, first the transition points $t p_{n}$ of the $m$-level Fresnel lens with focal length $f$ and for the wavelength $\lambda$ have to be calculated. The phase values $\varphi_{n}$ of the transmission function are thus given by

$\varphi_{n}=n \frac{2 \pi}{m}=\frac{2 \pi}{\lambda}\left[\left(f^{2}+t p_{n}^{2}\right)^{1 / 2}-f\right]+\varphi_{\mathrm{off}}$.

For reasons that are explained later and in section 4, we have introduced a constant offset phase $\varphi_{\text {off }}$ (Fig. 3). After solution of Eq. (4), we obtain for the transition points $t p_{n}$ 


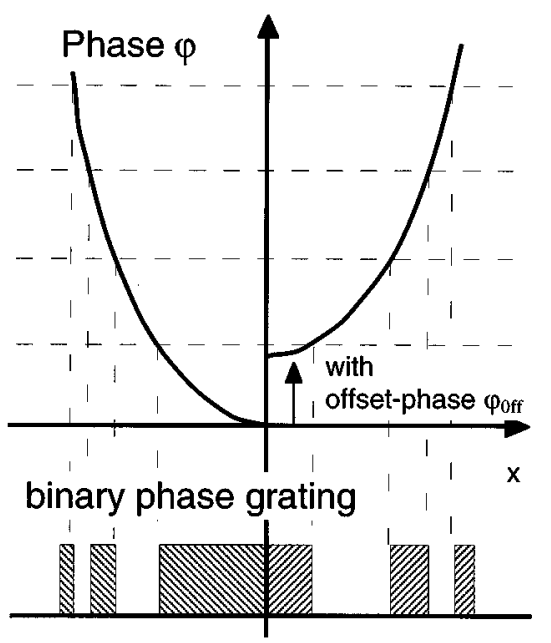

Fig. 3 Transition points of a binary phase grating for a parabolical phase function with (right) and without (left) offset phase.

$t p_{n}=\left[\left(\frac{n}{m}-\frac{\varphi_{\mathrm{off}}}{2 \pi}\right)^{2} \lambda^{2}+2\left(\frac{n}{m}-\frac{\varphi_{\mathrm{off}}}{2 \pi}\right) \lambda f\right]^{1 / 2}$

The contribution of the $n$ 'th zone to the far field in one dimension is given by

$U_{n}^{1 D}\left(x_{2}\right)=t p_{n} \frac{\sin \left(2 \pi t p_{n} x_{2}\right)}{2 \pi t p_{n} x_{2}}-t p_{n-1} \frac{\sin \left(2 \pi t p_{n-1} x_{2}\right)}{2 \pi t p_{n-1} x_{2}}$,

while for cylindrical symmetry the contribution can be written as

$U_{n}^{2 D}(\rho)=t p_{n}^{2} \frac{2 J_{1}\left(2 \pi \rho t p_{n}\right)}{2 \pi \rho t p_{n}}-t p_{n-1}^{2} \frac{2 J_{1}\left(2 \pi \rho t p_{n-1}\right)}{2 \pi \rho t p_{n-1}}$,

with $\rho=\left(x_{2}^{2}+y_{2}^{2}\right)^{1 / 2}$. Constant factors are neglected. The far field is obtained after summation of the single contributions, multiplied by the phase shift $\varphi_{n}$ of the corresponding zone according to Eq. (4):

$U^{1 D}\left(x_{2}\right)=\sum_{n=1}^{N} U_{n}^{1 D}\left(x_{2}\right) e^{i \varphi_{n}}$

or

$U^{2 D}(\rho)=\sum_{n=1}^{N} U_{n}^{2 D}(\rho) e^{i \varphi_{n}}$

The offset phase has a considerable influence to the first few contributions to the series in Eq. (8). The offset-phase defines the interference between these contributions. The first and second contributions, e.g., can be ajusted so that they are destructively interfering at $x_{2}=0($ or $\rho=0)$. The influence of the offset phase to the high frequencies is negligible.

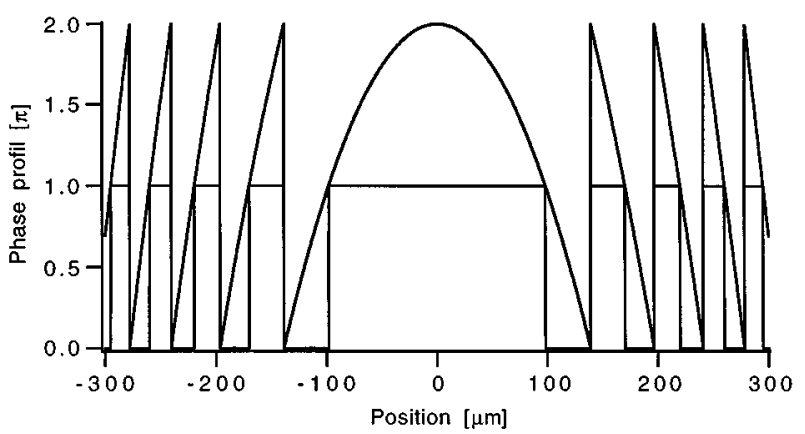

(a)

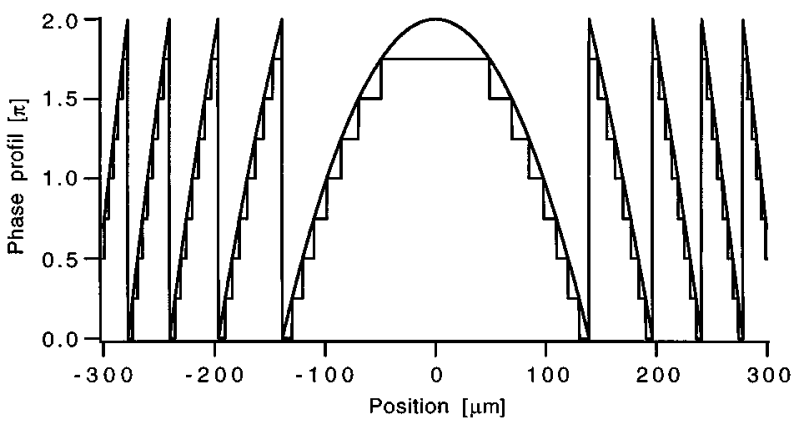

(b)

Fig. 4 Continuous phase function and (a) binary or (b) eight-level approximation.

We applied the algorithm, given in Eq. (5) to (8), to binary as well as multilevel gratings with and without offset phase. The results are given in the following sections.

\section{Effect of Offset Phase and Incoherent Illumination on the Far Field of Blazed Phase Gratings}

The phase grating or Fresnel zone lens is conventionally fabricated as a binary or multilevel phase grating. Due to the approximation of the continuous phase profile by a binary or staircase grating (Fig. 4), the far field shows rapid oscillations or binarization noise (Fig. 5). This noise is smoothed out by the incoherent illumination (Fig. 6). In addition, the center of the far field, rising from the center of the Fresnel zone lens, shows a deviating behavior. In the center of the zone plate, the approximation of the phase function especially by a binary phase grating is very poor [Fig. 4(a)]. The far field shows almost no variation in the center [Fig. 5(a)], giving rise to a large zeroth order [Fig. 6(a), divergency $1 \mathrm{mrad}]$.

Thus the optimization method for the zone plates to obtain homogeneous far-field distributions is obvious: either the large central peak must be reduced by approximating the ideal phase function by a multilevel phase grating [e.g., eight levels, Figs. 4(b), 5(b), and 6(b)] or the central part of the zone plate has to be disturbed. A disturbation of the central region can be obtained by an optimization of the central part of the zone plate only, e.g., by a different lens structure. As shown in Figs. 7 to 9, it is also sufficient to add just a constant offset phase to the parabolic or hyperbolic phase function. For a continuous profile in the thin- 


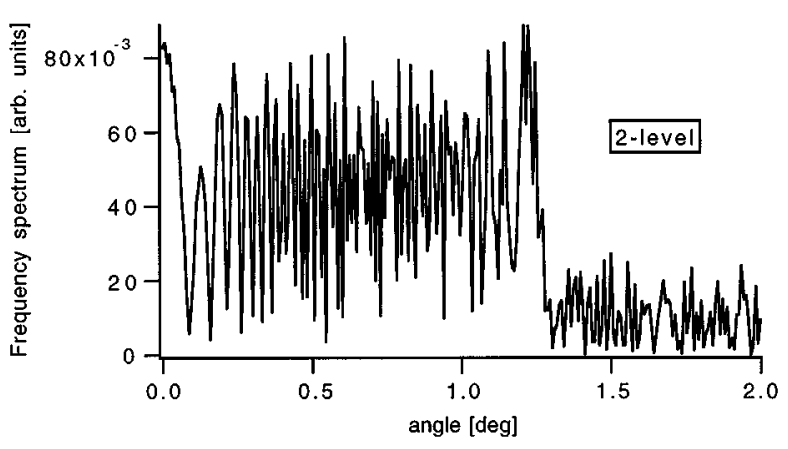

(a)

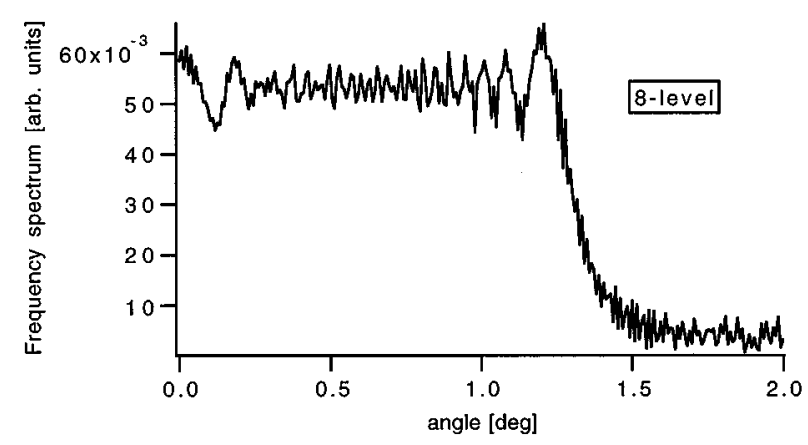

(b)

Fig. 5 Spectrum of (a) the binary and (b) the eight-level element, given in Fig. 4.

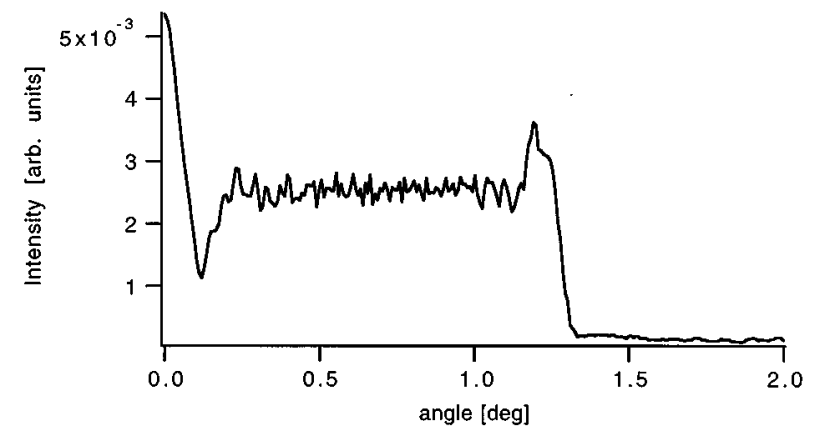

(a)

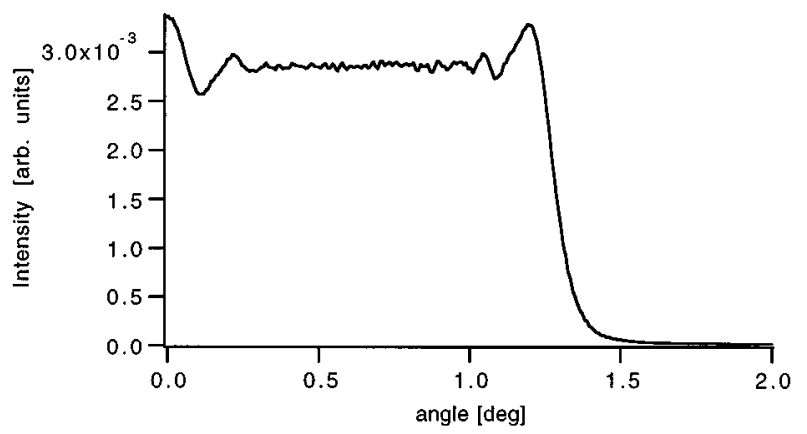

(b)

Fig. 6 Far-field distribution for incoherent illumination (divergency 1 mrad) for (a) the binary and (b) the eight-level element.

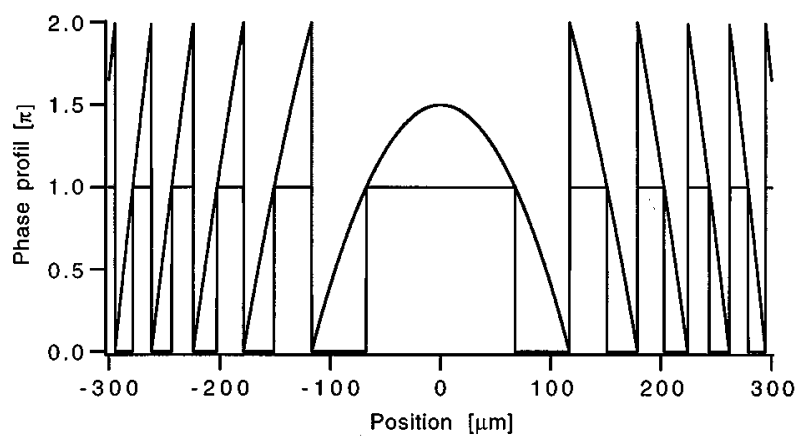

(a)

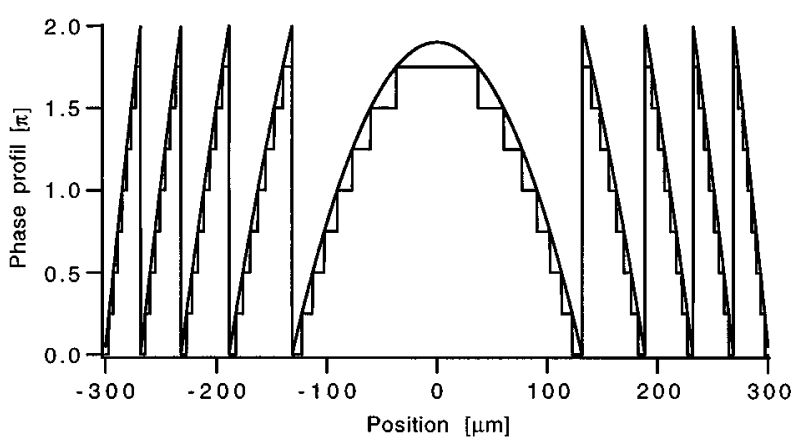

(b)

Fig. 7 Design of a (a) binary and (b) eight-level element with phase offset of (a) $0.45 \pi$ and (b) $0.1 \pi$.

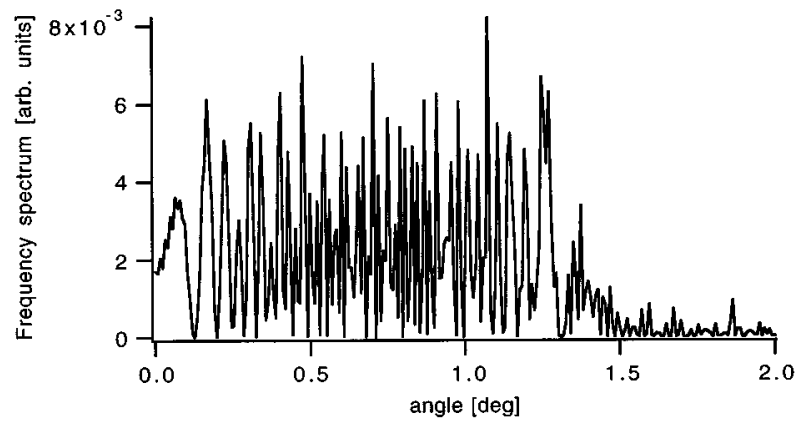

(a)

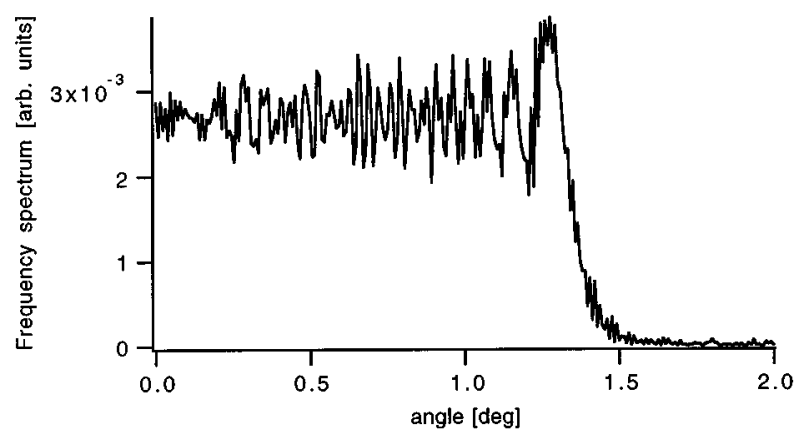

(b)

Fig. 8 Spectrum of (a) the binary and (b) the eight-level element with phase offset given in Fig. 7. 


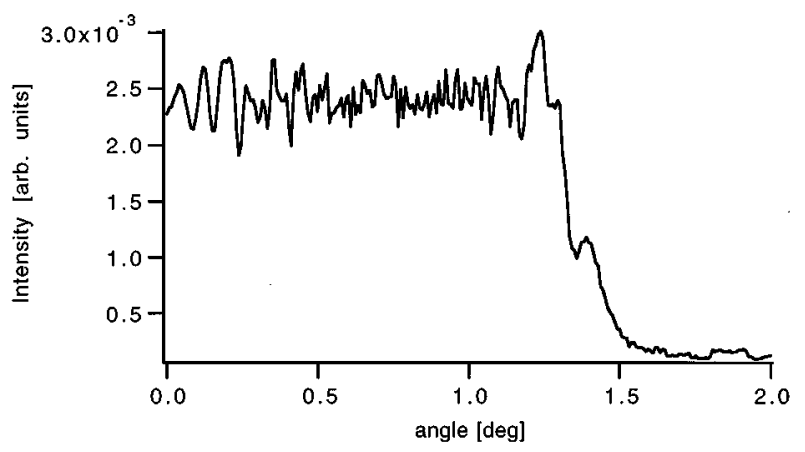

(a)

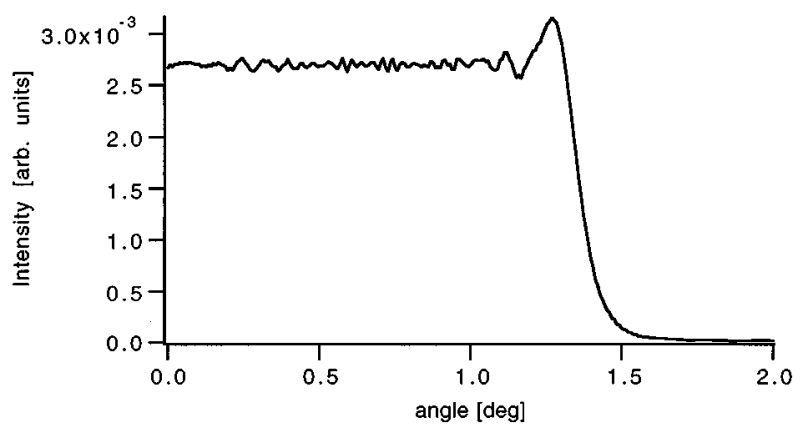

(b)

Fig. 9 Far-field distribution for incoherent illumination (divergency 1 mrad) for (a) the binary and (b) the eight-level element with offset phase.

element-approximation, this phase shift has no effect on the spectrum. However, for a binary phase grating, the central part is effected tremendously. The large central part of the zone plate in Fig. 4(a), which is not diffracting at all, is decreased [Fig. 7(a)]. The large unmodulated region in the spectrum of a zone plate [Fig. 8(a)] without phase offset is modulated. After convolution of the power spectrum with the spectrum of the incoherent source, the large zeroth orders vanish almost completely (Fig. 9). As demonstrated in Figs. 7(b) to 9(b), this method can also be applied to improve the homogenity of multilevel gratings.

From a comparison of the frequency spectrum for the binary grating with and without phase offset it can be seen that the difference is largest in the zeroth order and at the boundary of the spectrum. As can be easily verified by a comparison of Fig. 6 to Fig. 9, in this region the homogeneity of the far-field distributions is improved reasonably. For Figs. 7 to 9, the ideal offset phases were choosen ( $0.45 \pi$ for the binary, and $0.1 \pi$ for the eight-level grating). The ideal offset phases were obtained by varying the offset phase from 0 to $\pi$. With the ideal phase offsets, the size of the central zone of the diffractive element is approximately half of the size without the phase offset. For other phase values, the effect is scaling between the element with ideal phase offset and the ordinary Fresnel zone plate.

A further comparison is concerned with diffusors of different sizes, but with constant numerical aperture. Figure 10(a) gives the dependence of the number of Fresnel zones from the lens diameter for lenses with constant numerical aperture. The size of the central unmodulated region or the

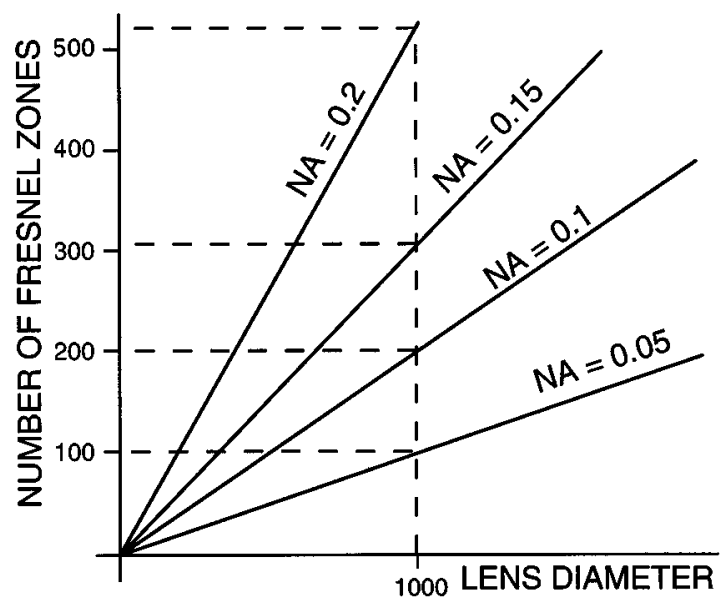

(a)

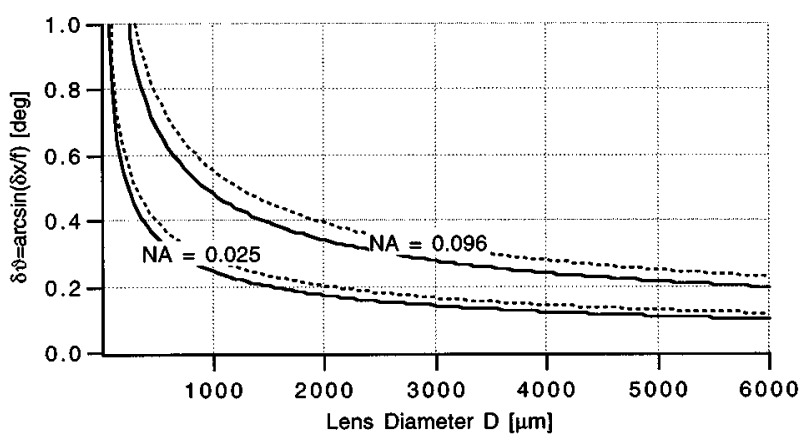

(b)

Fig. 10 (a) Number of Fresnel zones (values for $\lambda=248 \mathrm{~nm}$ ) and (b) maximum diffraction spot size (dashed line, with offset phase) in dependence of the lens diameter for constant numerical apertures.

first Fresnel zone according to Eq. (7) defines the maximum diffraction spot size in the center of the far-field distribution, as shown in Fig. 10(b). Figures 11 to 13 give the results of the 1-D analysis for different lens diameters $(500$ to $5000 \mu \mathrm{m}$ ) for binary Fresnel-zone lenses with an ideal offset phase of $0.45 \pi$. With the number of Fresnel zones, the number of contributions to the Fresnel sum [Eq. (8)] also increases. Therefore the frequency spectra exhibit an increasing modulation with increasing element size [Figs. 11 to 13(a)]. Especially the modulation frequency of the central part increases. Therefore the homogenity of the intensity distribution after convolution improves reasonably with increasing element size. However, with increasing element size of the micro-Frensel lenses, the space invariance of the diffusor is reduced. Therefore one has to treat the trade-off between homogenity of the intensity distribution and space invariance of the element.

Thus we have showed that by a simple addition of a constant offset phase to the continuous phase profile, the spectrum of the binary or multilevel phase grating can be modified. As already shown in Section 3, the effect of the offset phase can be interpreted as an interference effect from the different Fresnel zones. The offset phase design can especially be applied to improve the homogenity of the far-field distribution. To obtain a homogenous far-field dis- 


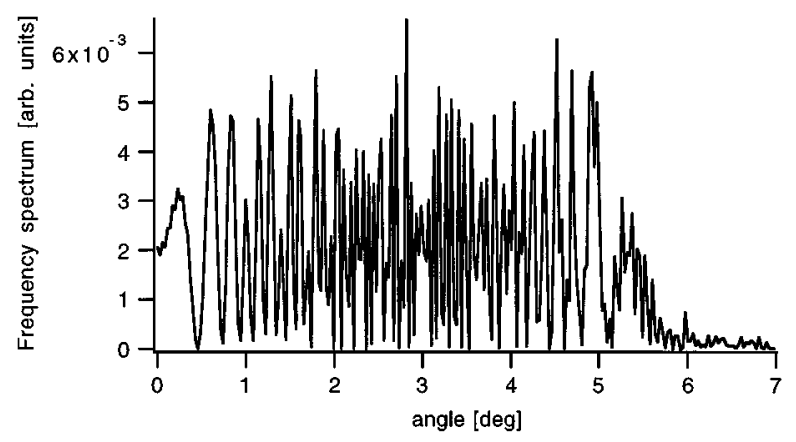

(a)

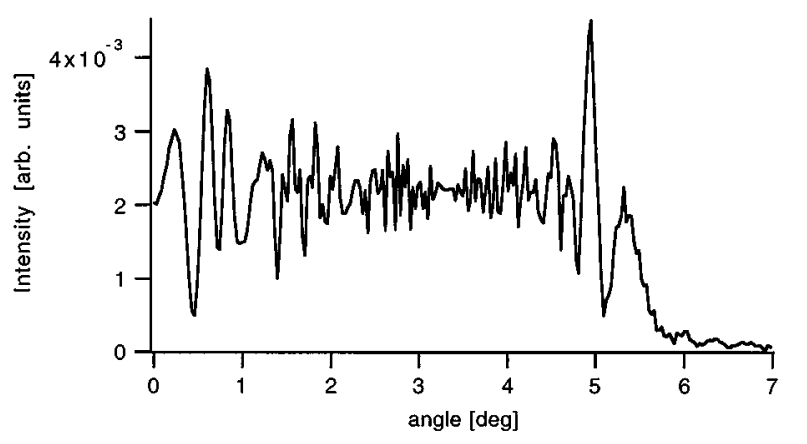

(b)

Fig. 11 (a) Frequency spectrum and (b) intensity distribution for incoherent illumination (1 mrad) for a binary Fresnel lens (diameter $500 \mu \mathrm{m})$.

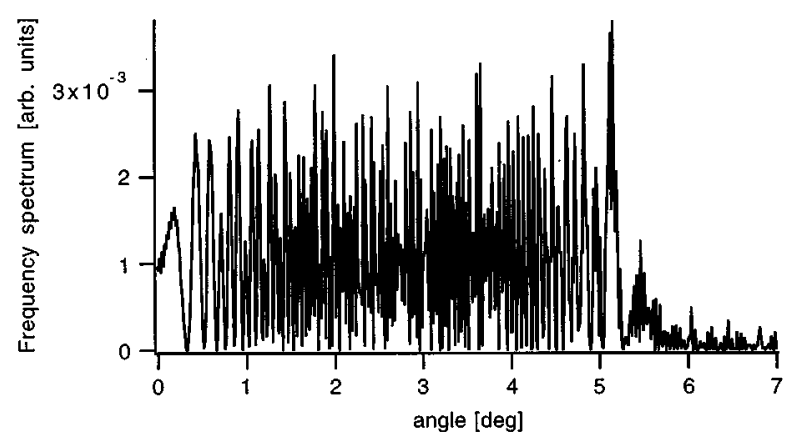

(a)

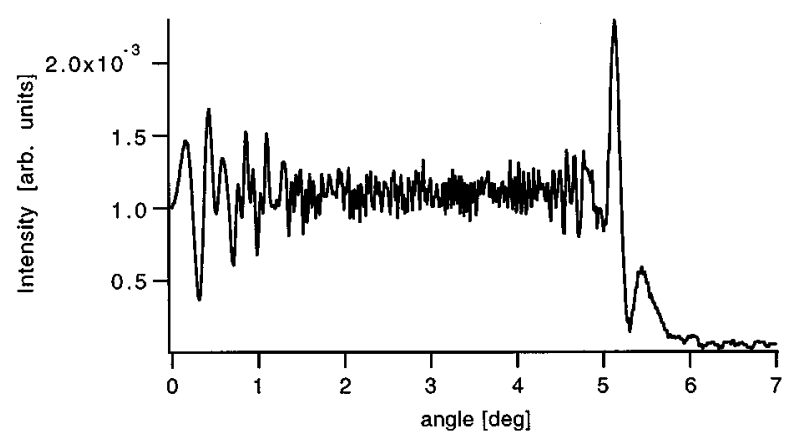

(b)

Fig. 12 (a) Frequency spectrum and (b) intensity distribution for incoherent illumination (1 mrad) for a binary Fresnel lens (diameter $1000 \mu \mathrm{m})$.

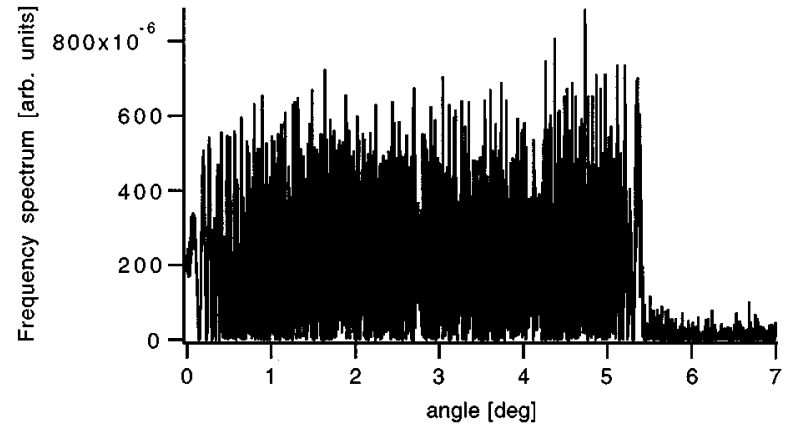

(a)

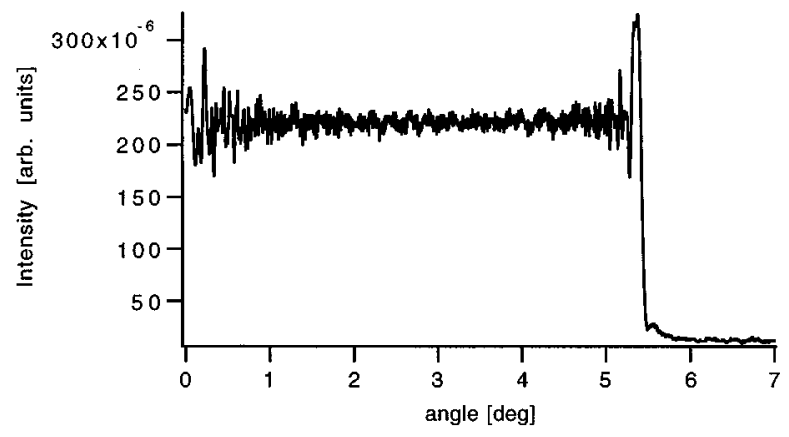

(b)

Fig. 13 (a) Frequency spectrum and (b) intensity distribution for incoherent illumination (1 mrad) for a binary Fresnel lens (diameter $5000 \mu \mathrm{m})$.

tribution with a given divergency angle of the incoherent source, a minimum element size must be choosen.

\section{Simulation Results and Comparison to Measurements}

A first design of a rectangular far-field distribution was performed with an array of rectangular binary Fresnel lenses without offset phase. The design angles were 4 and $5.5 \mathrm{deg}$ in $x$ and $y$. To consider the rectangular shape, the far-field distribution, calculated according Eq. (7), was convolved with a 1-D rect function. Therefore the spectrum could only be evaluated either in the $x$ or in $y$ direction. To consider the spherical symmetry, the convolution according to the divergency of the source was performed numerically without taking advantage of the Fourier property of the convolution. Results are shown for the $x$ direction in Fig. 14. In a second design, a Fresnel zone plate was fabricated with a constant offset phase. The simulation result and the measurement are compared in Fig. 15.

Both measurements show a significant deviation from the simulation result in the zeroth order due to fabrication errors, while the design angles (4 deg in Fig. 14 and 5.5 deg in Fig. 15) are obeyed. Etch depth errors occur due to deviations from the ideal etching parameters and inhomogenity effects. The diffraction efficiency of phase gratings is very sensible to etch depth errors, while the diffraction angles are not affected. A comparison of Figs. 14 and 15 demonstrates that nevertheless the zeroth order of the grat- 


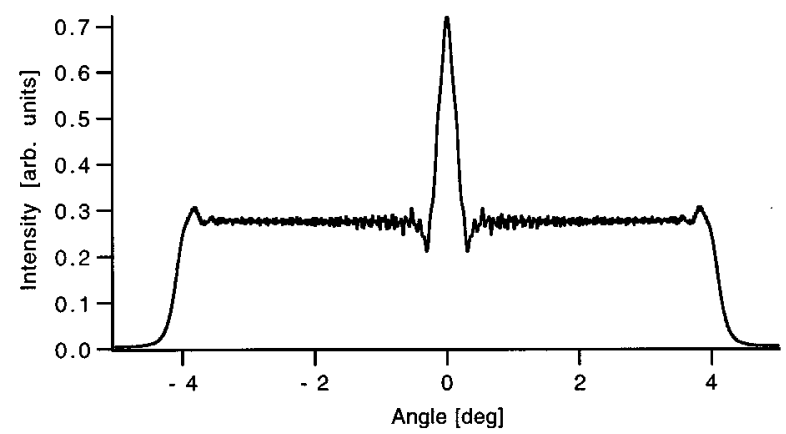

(a)

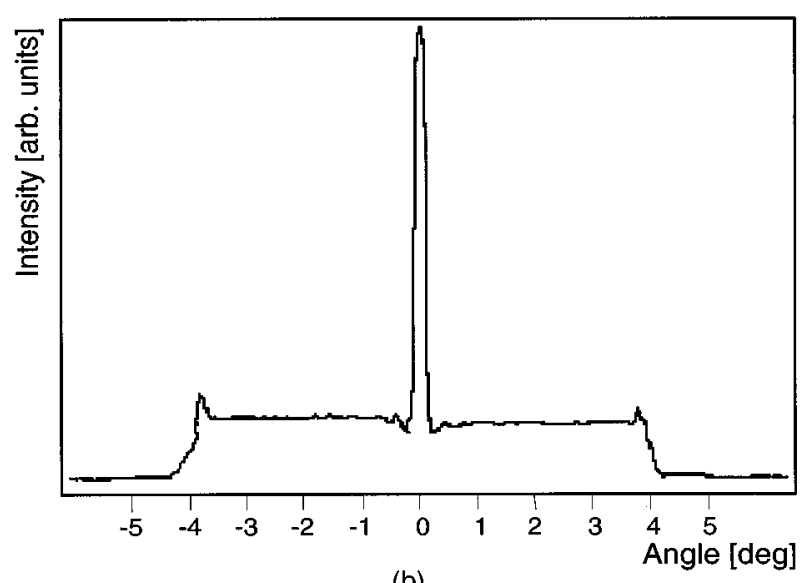

(b)

Fig. 14 (a) Simulation and (b) measurement result for a binary phase element without offset phase (design angle $4 \mathrm{deg}$ ).

ing with offset phase is smaller compared to the total efficiency than for the grating without phase offset.

Etch depth errors are therefore critical for the design of flat-top distributions. Therefore we investigate etch-depth errors more in detail in the following section.

\section{Etch Depth Errors}

Etch depth errors cause an increase of the zeroth diffraction order. Therefore etch depth errors destroy the homogenity of the flat-top distribution. The zeroth order increases with the area of the diffractive element. The result from a 2-D analysis shows therefore a larger increase of the peak-tovalley $(p-v)$ ratio with increasing etch depth error. The encircled energy in the central spot, however, shows for the 2-D evaluation the same behavior as the $p-v$ ratio for the $1-\mathrm{D}$ analysis. Thus the encircled energy increases by a factor of 4 from $\sim 0.05 \%$ to $\sim 0.2 \%$ for an etch-depth error of $5 \%$. The optimized grating with the offset phase (Fig. 16, dotted curve) shows the same behavior as the element without offset phase, i.e., the encircled energy in the zeroth order increases by the same factor.

Etching errors induce an enlargement of only the zeroth order. However, the total amount of energy in the central peak remains very small (in our application $<0.5 \%$ ). The fabrication tolerances of the etch depth are often below $5 \%$. Also the measurement result in Fig. 15 indicates an etch depth error of this size. Constant etch depth errors can in

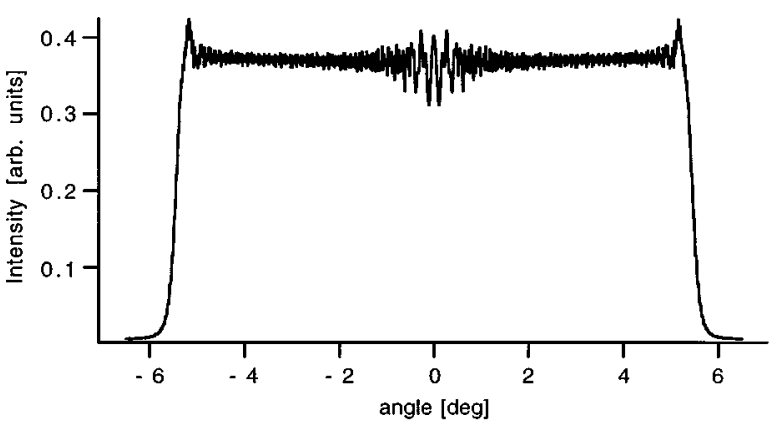

(a)

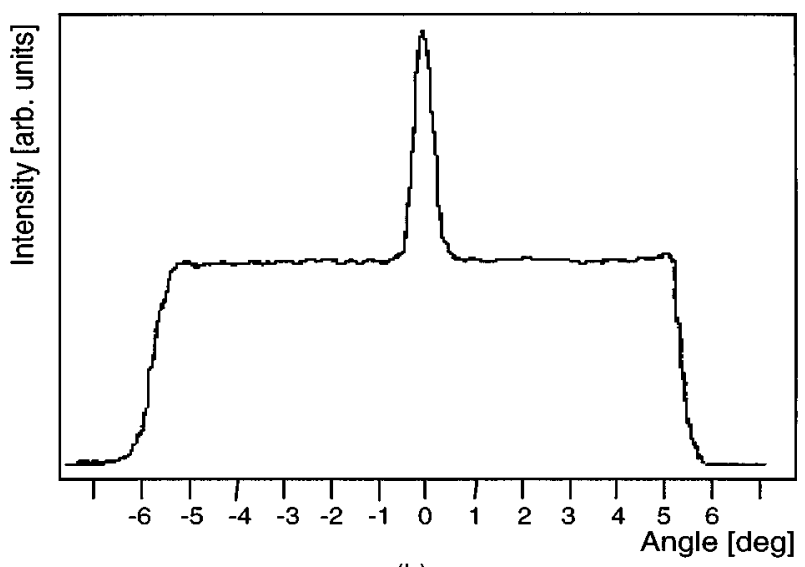

(b)

Fig. 15 (a) Simulation and (b) measurement result for a binary phase element with offset phase (design angle $5.5 \mathrm{deg}$ ).

principle be corrected by an optimization of the etching time or other parameters. In addition, however, the homogenity of the etch depth over the substrate might be insufficient. In this case, it is not possible to remove the zeroth order completely. To avoid the zeroth order, frequently an off-axis design is choosen. ${ }^{11}$ However, in our application this is not possible because of the fabrication limits. Therefore it is probably advantageous to defocus or to block out the zeroth order.

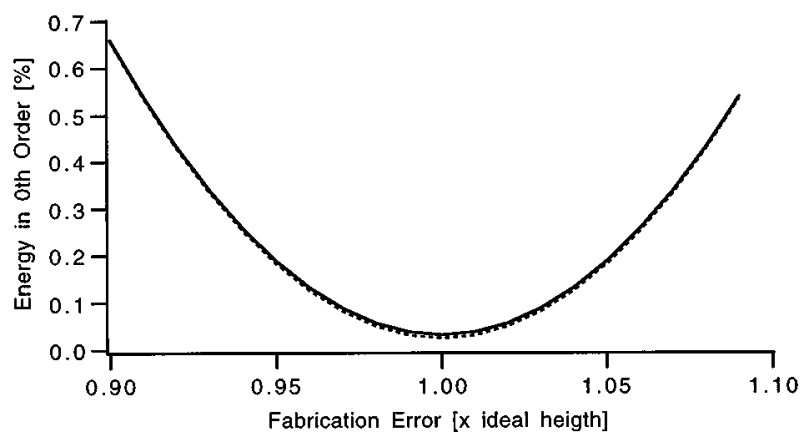

Fig. 16 Encirled energy in the central spot in dependence of etch depth errors, 2-D analysis. 


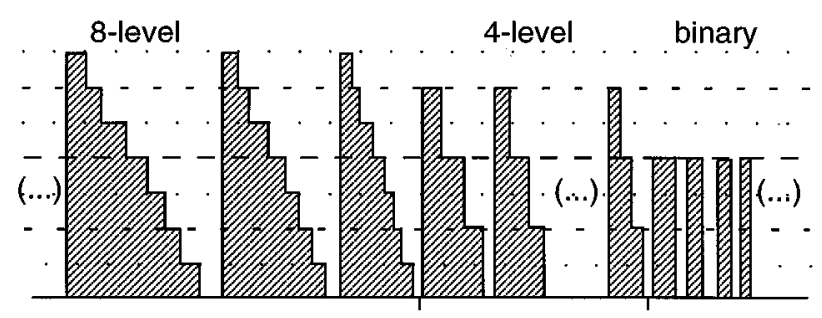

Fig. 17 Eight-, four-, two-level element, according to fabrication limits.

\section{Multilevel Phase Gratings at Small Wavelengths}

It is well known that the efficiency of multilevel or continuous phase gratings is larger compared to that of binary phase gratings. ${ }^{12}$ The efficiency of binary phase gratings is usually in the order of $40 \%$, which is improved, e.g., for eight-level elements to about 95\% (thin-element approximation). For diffusors with symmetrical far-field distributions, the +1 and the -1 diffraction order of the two-level elements contribute both to the far-field distribution. In this case, the diffration efficiency of binary elements is of the order of $80 \%$. However, the continuous transmission phase is better approximated with larger level numbers, decreasing the central intensity peak. For small wavelengths and large diffraction angles, the minimum feature size of an eight-level element might be beyond the resolution limit of the lithography. Thus, it might be not possible to fabricate an eight-level element over the entire aperture. We therefore investigated the optical properties of multilevel elements with varying level numbers. The central part of the Fresnel-zone plate with the small frequency is realized as an eight-level element, while the level number decreases with increasing frequency. Fig. 17 shows the design. In Fig. 18 the spectrum of an eight-, four-, two-level element is given. The different level numbers exhibit different binarization noise and are therefore easily distinguishable (Fig. 18).

After consideration of the incoherent illumination, the far-field distribution shows a step-like profile according to the diffraction efficiencies of the different number of grating levels (Fig. 19). Thus the efficiency is improved from $\sim 81$ to $\sim 87 \%$, but the homogenity of the far-field distribution is reduced. Therefore only binary elements are ap-

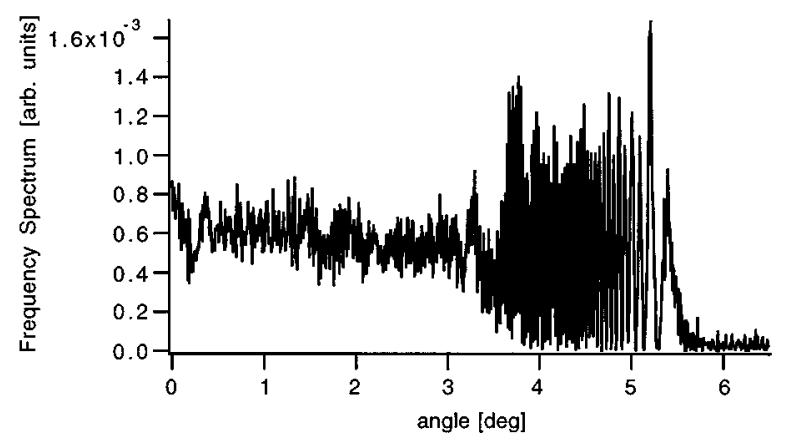

Fig. 18 Spectrum of the eight-, four-, two-level element.

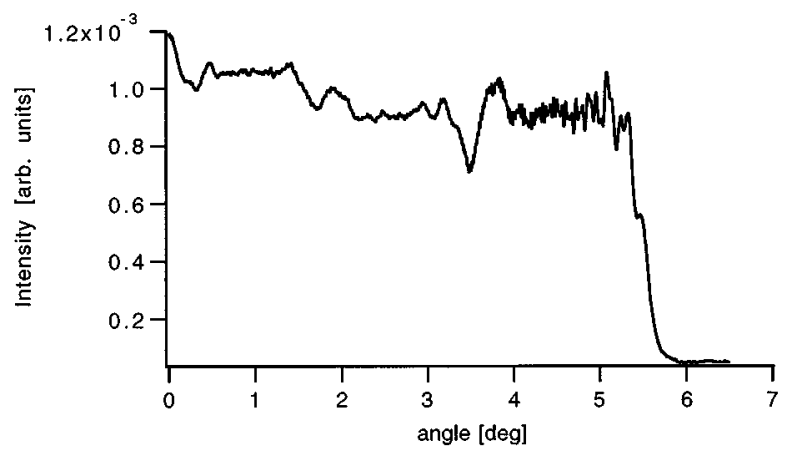

Fig. 19 Far-field distribution of an eight-, four-, two-level element with incoherent illumination (divergency $\sim 1 \mathrm{mrad}$ ).

plicable to the fabrication of homogeneous flat-top intensity distributions at small wavelengths and large angles (e.g., $5.5 \mathrm{deg}$ at $248 \mathrm{~nm})$.

\section{Conclusion}

In this paper, we discussed the generation of flat-top intensity distributions for excimer laser applications $(\lambda=248$ $\mathrm{nm}$ ) by diffractive phase gratings at the fabrication limit. Therefore we investigated the design and the optimization of micro-Fresnel lenses. The influence of an incoherent illumination as well as an constant offset phase on the farfield distribution of Fresnel zone plates was found to be applicable to improve the homogenity of the desired farfield distribution. We analyzed binary as well as multilevel gratings with and without offset phase by direct computation of the Fresnel series as well as by an FFT algorithm. In addition, we compared our results to measurements.

We showed in detail that by a simple addition of a constant offset phase to the continuous phase profile, the spectrum of the binary or multilevel phase grating can be modified. The offset phase design can especially be applied to improve the homogenity of the far-field distribution. The effect of the offset phase can be interpreted as a interference effect from the different Fresnel zones.

Etch depth errors and inhomogenities of the substrate induce an enlargement of the zeroth order. Therefore we found a large zeroth order from the measurements even for the optimized micro-Fresnel lens. However, the total amount of energy in the central peak remains very small and it is therefore advantageous to defocus or to block out the zeroth order.

With multilevel elements of varying level number, the efficiency is improved, but the homogenity of the far-field distribution is reduced. Therefore only binary elements are applicable to fabricate homogeneous flat-top intensity distributions at small wavelengths and large angles.

\section{Acknowledgments}

The authors are indebted to Peter Blattner for fruitful discussions. For the fabrication of the elements, Jean-Michel Mayor from the Centre Suisse d'Electronique et de Microtechnique SA (CSEM) (Neuchâtel) is acknowledged. This work was supported by Carl Zeiss, Oberkochen, Germany, and the Swiss Priority Program Optique. The authors are, however, solely responsible for the content. 


\section{References}

1. C. Budzinski and H.J. Tiziani, "Radiation resistant diffractive optics generated by micro electroforming,"' Laser Optoelektron. 27(1), 54-61 (1995).

2. T. H. Bett, R. M. Stevenson, M. J. Norman, C. N. Danson, D. A. Pepler, and I. N. Ross, "A binary phase zone plate array for laser beam spatial intensity distribution conversion," in Holographic Systems, Components and Applications, IEE Conference Publication 379 pp. 249-254, Neuchâtel (1993).

3. Joseph N. Mait, "Understanding diffractive optic design in the scalar domain," J. Opt. Soc. Am. A 12, 2145-2158 (1995).

4. H. Nishihara and T. Suhara, "Micro Fresnel Lenses,' in Progress in Optics, Vol. 24, p. 4, Elsevier (1987).

5. E. Hasman, N. Davidson and A. A. Friesem, "Efficient multilevel phase holograms for $\mathrm{CO}_{2}$-Lasers," Opt. Lett. 16, 423-425 (1991).

6. J. W. Goodman, Introduction to Fourier Optics, chap. 6, pp. 110-120 McGraw-Hill, New York (1968).

7. J. W. Goodman, Introduction to Fourier Optics, chap. 5, pp. 77-83 McGraw-Hill, New York (1968)

8. K. Iizuka, Engineering Optics, Springer Series in Optical Sciences Vol. 35, 6th ed., chap. 7, pp. 169-185, Springer-Verlag, New York (1987).

9. A. E. Siegman, "Quasi fast Hankel transform," Opt. Lett. 1(1), 13-15 (1977).

10. I. N. Ross, D. A. Pepler, and C. N. Danson, "Binary phase zone plate design using calculations of far-field distributions," Opt. Commun. 116, 55-61 (1995); M. Born and E. Wolf, Principles of Optics, 6th ed., pp. 395-398, Pergamon Press, Oxford (1980).

11. H. Ichikawa, J. Turunen and M. R. Taghizadeh, "Analysis of hybrid gratings by thin-grating decomposition method," J. Opt. Soc. Am. A 10, 1176-1183 (1993).

12. J. W. Goodman and A. M. Silvestri, "Some effects of Fourier-domain phase quantization,' IBM J. Res. Develop. 14, 478-484 (1970).

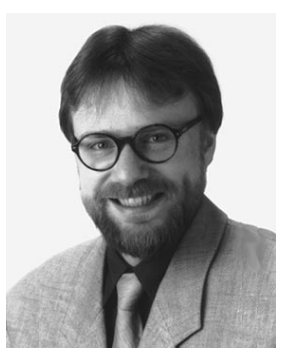

Wolfgang Singer received his MS degree from the Department of Applied Optics, University of Erlangen, Nuernberg, in 1991 and finished his $\mathrm{PhD}$ in the field of gradient index optics in 1995. He then spent 8 months at the Institute of Microtechnology in Neuchâtel, Switzerland, as a research fellow. Since 1996 he has been a research assistant at the Institut fuer Technische Optik, Stuttgart.

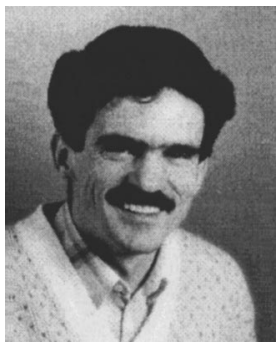

Hans Peter Herzig received the diploma in physics from the Swiss Federal Institute of Technology in Zürich (ETHZ), Switzerland, in 1978. From 1978 to 1982 he was a scientist in the Optics Development Department of the company Kern in Aarau, Switzerland, where he worked in lens design and optical testing. In 1983, he joined the Applied Optics Group at the Institute of Microtechnology of the University of Neuchâtel, Switzerland, as a graduate research assistant, working in the field of holographic optical ele- ments, especially scanning elements. In 1987, he received a PhD degree in optics from the University of Neuchâtel. Currently, he is lecturing in modern optics and is responsible for the research in micro-optics, including diffractive optical elements and microlenses.

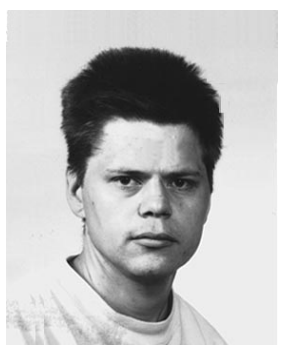

Markku Kuittinen received MS and LicPhil degrees in physics from the University of Kuopio in 1989 and 1992, respectively, and a PhD degree in physics from the University of Joensuu in 1994, after which he spent 1 year as a visiting researcher at the Institute of Microtechnology in Neuchâtel, Switzerland, where he is currently a researcher in the Department of Physics. His research interests are in the area of numerical simulation and design and optimization of diffractive optical elements.

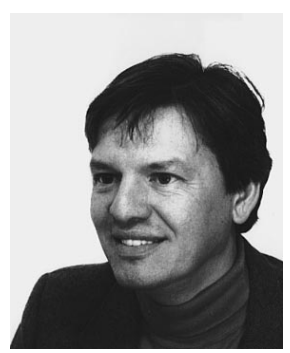

Eckhard Piper graduated from the Technical University of Hannover in Germany with a DiplPhys in 1978. He joined the Max-Planck Institut für Strömungsforschung in Göttingen and received his $\mathrm{PhD}$ degree from the University of Göttingen in 1983. From 1984 to 1989 he worked for AEG Research Center as a member of the X-Ray Lithography Group at the BESSY Electron Storage Ring in Berlin. Since 1990 he has been working for Zeiss on the development of UV and deep UV illumination systems.

Johannes Wangler: Biography and photograph not available. 